\title{
THE FARRELL-JONES CONJECTURE FOR ARBITRARY LATTICES IN VIRTUALLY CONNECTED LIE GROUPS
}

\author{
HOLGER KAMMEYER, WOLFGANG LÜCK, AND HENRIK RÜPING
}

\begin{abstract}
We prove the $K$ - and the $L$-theoretic Farrell-Jones conjecture with coefficients in additive categories and with finite wreath products for arbitrary lattices in virtually connected Lie groups.
\end{abstract}

\section{INTRODUCTION}

The Farrell-Jones conjecture predicts the algebraic $K$-theory and $L$-theory of group rings. The original formulation can be found in Farrell-Jones [13. We will deal with the more general version with coefficients in additive categories and with finite wreath products, see [3,8, and 7, Definition 6.1]. The relevance of the Farrell-Jones Conjecture comes from the fact that it implies many other prominent conjectures, for instance the one due to Borel about topological rigidity of aspherical closed manifolds, the one due to Novikov about the homotopy invariance of higher signatures, and the one due to Kaplansky about the triviality of idempotents in group rings with coefficients in a field of torsionfree groups. For an overview of the Farrell-Jones conjecture and its consequences, see for instance [5, 20,21.

Let $\mathfrak{F} \mathfrak{J}$ be the class of groups satisfying the K- and L-theoretic Farrell-Jones conjecture with coefficients in additive categories and with finite wreath products. Recently, the third named author 28 proved $\mathrm{GL}(n, \mathbb{Q}) \in \mathfrak{F} \mathfrak{J}$. In this article we show that the theory of deformations and rigidity of lattices in semisimple Lie groups due to Calabi, Vesentini and Weil allow the following conclusion. A topological group is called virtually connected if it has finitely many path components. A discrete subgroup of a locally compact Hausdorff group is called a lattice if the quotient space $G / \Gamma$ has finite covolume with respect to the Haar measure of $G$.

Theorem 1 (Lattices in virtually connected Lie groups). Let $G$ be a virtually connected Lie group and let $\Gamma \subset G$ be a lattice.

Then $\Gamma$ lies in $\mathfrak{F} \mathfrak{J}$.

This extends a previous result of Bartels, Farrell and the second named author 2 from the class of cocompact lattices to the class of all lattices in virtually connected Lie groups.

Deligne-Mostow [12] have constructed non-cocompact lattices in $\mathrm{SU}(2,1)$ and $\mathrm{SU}(3,1)$ which are neither hyperbolic, nor $\mathrm{CAT}(0)$, nor arithmetic, nor solvable (not even amenable). So Theorem 1 comprises groups for which the Farrell-Jones conjecture was a priori unknown. Note that the operator-theoretic version of the Farrell-Jones Conjecture, the Baum-Connes conjecture for the topological $K$-theory of the reduced group $C^{*}$-algebra, is still open for many lattices in virtually connected Lie groups, for instance for $S L(n, \mathbb{Z})$ for $n \geq 3$.

Date: January 2014

2010 Mathematics Subject Classification. Primary: 18F25, 19A31, 19B28, 19G24, 22A26.

Key words and phrases. Farrell-Jones Conjecture, lattices in virtually connected Lie groups. 
The in our view most general result about lattices will be proved in Theorem 8 . where the virtually connected Lie group is replaced by a second countable locally compact Hausdorff group.

This paper has been financially supported by the Leibniz-Award, granted by the Deutsche Forschungsgemeinschaft, of the second named author.

\section{The status of the Farrell-Jones Conjecture}

The next result describes what is known about $\mathfrak{F} \mathfrak{J}$. We will frequently use some of the properties of $\mathfrak{F} \mathfrak{J}$ listed below, no more knowledge about the Farrell-Jones Conjecture is required to understand the proofs in this paper.

Theorem 2 (Status of the Farrell-Jones Conjecture). The class of groups $\mathfrak{F} \mathfrak{J}$ has the following properties:

(1) It contains all hyperbolic groups and all CAT(0)-groups;

(2) It contains all solvable groups;

(3) It contains $\mathrm{GL}(n, \mathbb{Q})$ and $\mathrm{GL}(n, F(t))$ for any finite field $F$;

(4) It contains all $S$-arithmetic groups;

(5) It contains all cocompact lattices in virtually connected Lie groups;

(6) It contains the fundamental group of any manifold of dimension $\leq 3$;

(7) It is closed under direct and free products;

(8) It is closed under taking subgroups;

(9) If $f: G \rightarrow H$ is a group homomorphism such that $H$, $\operatorname{ker} f$ and $f^{-1}(Z)$ lie in $\mathfrak{F} \mathfrak{J}$ for every infinite cyclic subgroup $Z \subset H$, then $G$ lies in $\mathfrak{F} \mathfrak{J}$;

(10) If a finite index subgroup of a group $G$ lies in $\mathfrak{F} \mathfrak{J}$, so does $G$;

Proof. See for instance [2, 4, 6, 28, 32, 33] for proofs (without wreath products). The version and the corresponding proofs with wreath products are just a slight modification of the version without wreath products, compare [2, Remark 0.4].

Farrell and Jones [14, Proposition 0.10] have proved the $L$-theoretic Farrell-Jones Conjecture and the $K$-theoretic Farrell-Jones Conjecture in dimensions $\leq 1$, both with untwisted coefficients in $\mathbb{Z}$, for fundamental groups of A-regular negatively curved complete Riemannian manifolds.

A Riemannian manifold is $A$-regular if for some nonnegative sequence $A=(A)_{i}$ we have $\left|\nabla^{i} R\right| \leq A_{i}$ where the indices i vary over the natural numbers and $\nabla^{i} R$ is the $i$-th covariant derivative of the curvature tensor.

If the lattice is torsionfree, then the quotient of the symmetric space by the action of that lattice is an $A$-regular manifold. We want to also include lattices with torsion and allow twisted coefficients and want to consider all degrees. This makes the use of the important inheritance properties of the general version possible.

\section{Some PRELIMINARIES ABOUT FINITELY GENERATED DISCRETE SUBGROUPS IN LINEAR ALGEBRAIC GROUPS DEFINED OVER $\mathbb{Q}$}

A key ingredient in our proof is the following striking property of lattices whose first cohomology with coefficients in the adjoint representation vanishes [26, Proposition 6.6 and Theorem 6.7, pp. 90-91].

Theorem 3. Let $\mathbf{G}$ be a linear algebraic group defined over $\mathbb{Q}$. If $\Gamma \subset \mathbf{G}(\mathbb{R})$ is a finitely generated discrete subgroup and $H^{1}(\Gamma, \mathfrak{g})=0$, then there exists a number field $\mathbb{F}$ and an element $g \in \mathbf{G}(\mathbb{R})$ such that $g \Gamma g^{-1} \subset \mathbf{G}(\mathbb{F})$.

We want to give an idea as to why group cohomology decides about the possibility of conjugating a lattice into the $\mathbb{F}$-rational points of an algebraic group. For more information, see [31, Section 6]. Let $G=\mathbf{G}(\mathbb{R})$ and let $\operatorname{Hom}(\Gamma, G)$ be the space 
of all homomorphisms from $\Gamma$ to $G$ with the topology of pointwise convergence. A base point $u \in \operatorname{Hom}(\Gamma, G)$ is given by the inclusion $u: \Gamma \hookrightarrow G$. A deformation of the lattice $\Gamma$ in $G$ is a map $\varphi: I \rightarrow \operatorname{Hom}(\Gamma, G)$, defined on some open interval $I$ containing zero, such that $\varphi_{0}=u$ and such that $\varphi(\gamma): I \rightarrow G$ is smooth for every $\gamma \in \Gamma$. Given a smooth path $g: I \rightarrow G$ with $g_{0}=e \in G$, we obtain a deformation setting $\varphi(g)_{t}=g_{t} u g_{t}^{-1}$. These deformations are always present, regardless of the specific group $\Gamma$ and its embedding $u$ in $G$. Therefore the deformations $\varphi(g)$ are termed trivial. Right multiplication with an element $h \in G$ defines a self-diffeomorphism $R(h)$ of $G$. We identify the Lie algebra $\mathfrak{g}$ of $G$ with the tangent space $T_{e}(G)$. Then any deformation $\varphi$ defines a function $c(\varphi): \Gamma \rightarrow \mathfrak{g}$ setting

$$
c(\varphi)(\gamma)=\left.\mathrm{d} R\left(\gamma^{-1}\right) \frac{\mathrm{d}}{\mathrm{d} t} \varphi(\gamma)_{t}\right|_{t=0} .
$$

One easily verifies that $c(\varphi)$ is a cocycle of $\Gamma$ with values in $\operatorname{Ad} \circ u: \Gamma \rightarrow \mathfrak{g}$. For a smooth path $g: I \rightarrow G$ with $g_{0}=e$ let $X_{g} \in \mathfrak{g}$ be the velocity vector of $g$ at $t=0$. Then for the trivial deformation $\varphi(g)$ we obtain $c(\varphi(g))(\gamma)=X_{g}-$ Ad $\circ$ $u(\gamma) X_{g}$ which means $c(\varphi(g))$ is a coboundary. This lets one hope that the condition $H^{1}(\Gamma, \mathfrak{g})=0$ might imply that every deformation of $\Gamma$ in $G$ is trivial. Indeed, this can be proved using, amongst other things, the implicit function theorem.

Let $\gamma_{1}, \ldots, \gamma_{n}$ be a choice of generators of $\Gamma$. Then we obtain an embedding $\operatorname{Hom}(\Gamma, G) \rightarrow G^{n}$ by sending a homomorphism $r \in \operatorname{Hom}(\Gamma, G)$ to the $n$-tuple $\left(r\left(\gamma_{1}\right), \ldots, r\left(\gamma_{n}\right)\right)$. We write each relation of $\Gamma$ as a word $w=w\left(\gamma_{1}, \ldots, \gamma_{n}\right)$ in the symbols $\gamma_{i}$. Allowing general elements $g \in G$ to take the place of the $\gamma_{i}$, each relation $w$ defines a morphism $w: G^{n} \rightarrow G$ of real affine varieties defined over $\mathbb{Q}$. The image of our embedding $\operatorname{Hom}(\Gamma, G) \rightarrow G^{n}$ is then given by $\bigcap_{w} w^{-1}(e)$, the intersection taken over all relations in $\Gamma$. Thus $\operatorname{Hom}(\Gamma, G)$ is embedded as a $\mathbb{Q}$ subvariety of $G^{n}$, no matter whether finitely many relations are sufficient or not. If $H^{1}(\Gamma, \mathfrak{g})=0$, then every deformation of $\Gamma$ in $G$ is trivial. So in that case the orbit of $u$ in $\operatorname{Hom}(\Gamma, G)$ under the $G$-action by conjugation contains an open neighborhood $U \subset \operatorname{Hom}(\Gamma, G)$ of $u$ which also implies that the point $u$ is simple. It is then a lemma of algebraic geometry [16, Lemma 7.1, p. 311] that $U$ contains a $\overline{\mathbb{Q}}$-rational point $u^{\prime}$ where $\overline{\mathbb{Q}}$ is the algebraic closure of $\mathbb{Q}$. Since $u^{\prime}$ is an $n$-tuple of matrices, clearly $u^{\prime}$ is in fact an $\mathbb{F}$-rational point for a finite extension $\mathbb{F}$ of $\mathbb{Q}$. This gives the theorem. It remains the question which lattices $\Gamma$ have vanishing $H^{1}(\Gamma, \mathfrak{g})$.

Let $G$ be a connected semisimple Lie group. The Lie algebra $\mathfrak{g}$ of $G$ has a decomposition $\mathfrak{g}=\mathfrak{g}_{1} \oplus \cdots \oplus \mathfrak{g}_{k}$ into simple ideals which is unique up to permutation. The unique connected Lie subgroup $G_{i}$ in $G$ with Lie subalgebra $\mathfrak{g}_{i}$ is a priori not closed. But since $\mathfrak{g}_{i}$ is an ideal, the group $G_{i}$ is normal and hence actually closed by [27. Multiplication defines an epimorphism

$$
G_{1} \times \cdots \times G_{k} \rightarrow G
$$

with discrete (in fact central) kernel. This is equivalent to $G$ being the almost direct product of the normal subgroups $G_{i}$, i.e., $G=G_{1} \cdot G_{2} \cdots G_{k}:=\left\{g_{1} \cdot g_{2} \cdots g_{k} \mid\right.$ $\left.g_{i} \in G_{i}, i=1,2, \ldots, k\right\}$ and the intersection of $G_{i}$ with $\prod_{j \neq i} G_{j}$ is discrete for all $i$. A compact factor $K$ of $G$ is a connected, normal, compact subgroup of $G$. It follows from [27] that $K$ is an almost direct product $K=G_{i_{1}} \cdots G_{i_{l}}$ with $1 \leq i_{1}<\cdots<i_{l} \leq k$ and each $G_{i_{j}}$ compact.

Thus a connected semisimple Lie group without compact factors is a connected semisimple Lie group all of whose connected, normal, compact subgroups are trivial. We remark that there is an equivalent definition in the literature requiring instead that all compact quotient groups of $G$ are trivial.

Following [26. Definition 5.20, p. 86], we call a lattice $\Gamma \subset G$ irreducible if there are no two normal, connected, infinite subgroups $H_{1}, H_{2} \subset G$ such that $G$ is the 
almost direct product of $H_{1}$ and $H_{2}$ and such that $\left(\Gamma \cap H_{1}\right) \cdot\left(\Gamma \cap H_{2}\right)$ has finite index in $\Gamma$.

Theorem 4. Let $G$ be a connected semisimple Lie group without compact factors and let $\Gamma \subset G$ be an irreducible lattice. Suppose that $G$ is not locally isomorphic to $\operatorname{SL}(2, \mathbb{R})$ or $\operatorname{SL}(2, \mathbb{C})$. Then $H^{1}(\Gamma, \mathfrak{g})=0$.

Proof. Since the group $G_{i}$ appearing in (10) is compact if and only if $G_{i}$ has real rank zero, we see that a rank one semisimple Lie group without compact factors is actually simple. Therefore Theorem 4 is a combination of [31, Corollary 7.5 and Theorem 7.7, p. 99].

\section{Proof of Theorem 1}

We first prove the semisimple case of Theorem 1 and then show how to deduce the result in general.

Proposition 5. Let $G$ be a connected semisimple Lie group and let $\Gamma \subset G$ be a lattice. Then $\Gamma \in \mathfrak{F} \mathfrak{J}$.

Proof. Let $K \subset G$ be the maximal compact factor. Consider the short exact sequences

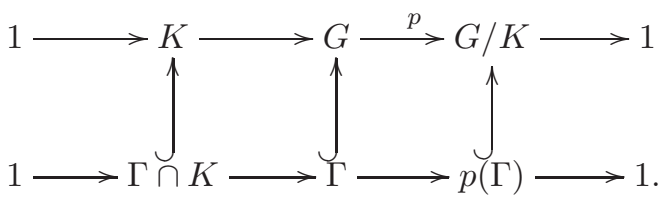

By [31, Corollary 4.10, p. 24] the image $p(\Gamma)$ of $\Gamma$ is a lattice in $G / K$. The group $\Gamma \cap K$ is finite because $K$ is compact and $\Gamma$ is discrete. Thus any preimage of a virtually cyclic subgroup is again virtually cyclic and hence lies in $\mathfrak{F} \mathfrak{J}$, for example by Theorem 2 (1). It follows from Theorem 2 (9) that the lattice $\Gamma$ lies in $\mathfrak{F} \mathfrak{J}$ if $p(\Gamma)$ does. Thus we may assume that $G$ has no compact factors.

Let $Z(G)$ denote the center of $G$. It follows now from [26, Corollary 5.17, p. 84] that the product $Z(G) \Gamma$ is discrete and in particular closed in $G$ because $G$ is a Hausdorff group. Since the center $Z(G)$ is a normal subgroup, we obtain from [26, Theorem 1.13, p. 23] that $\Gamma \cap Z(G)$ is a lattice in $Z(G)$, which in this case just means it has finite index. Thus [24, Theorem 4.7, p. 23] says that $\Gamma$ projects under $p: G \rightarrow G / Z(G)$ to a lattice $p(\Gamma) \subset G / Z(G)$. Moreover, the center $Z(G)$ is an abelian group so that any preimage under $p$ of a virtually cyclic subgroup in $G / Z(G)$ is virtually solvable, thus lies in $\mathfrak{F} \mathfrak{J}$ by Theorem 2 (2) and (10). Again by Theorem 2 (9) we may assume that $G$ has trivial center.

We conclude from [26, Theorem 5.22, p. 86] that we have an almost direct product decomposition $G=H_{1} \cdots H_{r}$ such that the almost direct product $\Gamma_{1} \cdots \Gamma_{r}$ has finite index in $\Gamma$ where $\Gamma_{i}=\Gamma \cap H_{i}$ is an irreducible lattice in $H_{i}$ for each $i=1, \ldots, r$. Recall that discrete normal subgroups of connected topological groups are central. Since $G$ has trivial center, both almost direct products are actually direct products. Thus by Theorem 2 (7) and (8) we can assume that $\Gamma$ is an irreducible lattice in a connected semisimple Lie group $G$ with trivial center and without compact factors.

Suppose $G$ was locally isomorphic to $\operatorname{SL}(2, \mathbb{R})$ or $\operatorname{SL}(2, \mathbb{C})$. Since $G$ has trivial center, $G$ is actually globally isomorphic to $\operatorname{PSL}(2, \mathbb{R})$ or $\operatorname{PSL}(2, \mathbb{C})$. Thus $\Gamma$ acts properly with finite volume quotient on hyperbolic 2- or 3-space. Therefore [10, Corollary 11.28, p. 362] asserts that $\Gamma$ is $\operatorname{CAT}(0)$ whence in $\mathfrak{F} \mathfrak{J}$ by Theorem 2 (1). So we may assume that $G$ is neither locally isomorphic to $\operatorname{SL}(2, \mathbb{R})$ nor to $\operatorname{SL}(2, \mathbb{C})$. By Theorem 4 we then have $H^{1}(\Gamma, \mathfrak{g})=0$. Moreover, the lattice $\Gamma \subset G$ is finitely generated, see [26, Remark 13.21, p. 210]. 
Let $\mathfrak{g}$ be the Lie algebra of $G$. The adjoint representation Ad: $G \rightarrow \operatorname{Aut}(\mathfrak{g})$ embeds $G$ as the Lie subgroup of inner automorphisms $\operatorname{Int}(\mathfrak{g}) \subset \operatorname{Aut}(\mathfrak{g})$ as is shown in [19, 5.2.(ii), p. 129]. Since $\mathfrak{g}$ is semisimple, the $\operatorname{subgroup} \operatorname{Int}(\mathfrak{g})$ is actually just the identity component of $\operatorname{Aut}(\mathfrak{g})$ by [19, Corollary 6.5, p. 132]. In addition it is well-known that a real semisimple Lie algebra admits a basis with rational structure constants [9, Proposition 3.7, p. 118]. It follows that $\mathbf{G}=\operatorname{Aut}(\mathfrak{g}) \subset \operatorname{GL}(\mathfrak{g})$ is a linear algebraic $\mathbb{Q}$-group and $G=\mathbf{G}(\mathbb{R})^{0}$.

Finally, Theorem 3 asserts that $\Gamma$ is conjugate to a subgroup of the $\mathbb{F}$-rational points $\mathbf{G}(\mathbb{F})$ for a number field $\mathbb{F}$. By restriction of scalars [25, Section 2.1.2] there exists a linear algebraic $\mathbb{Q}$-group $\operatorname{res}_{\mathbb{F} / \mathbb{Q}}(\mathbf{G})$ such that $\mathbf{G}(\mathbb{F})=\operatorname{res}_{\mathbb{F} / \mathbb{Q}}(\mathbf{G})(\mathbb{Q}) \subset$ $\mathrm{GL}(n, \mathbb{Q})$. Theorem 2 (3) and (8) completes the proof.

Remark 6 . The above proof starts by showing that we can assume the Lie group $G$ is connected, semisimple, center-free and has no compact factors while the lattice $\Gamma$ is irreducible. If one additionally requires that $G$ has real rank at least two, then the assumptions of Margulis' famous arithmeticity theorem [22, Theorem 1] are satisfied which says in particular that $\Gamma$ virtually embeds into $\operatorname{GL}(n, \mathbb{Z})$. The Farrell-Jones conjecture for $\Gamma$ then follows from 7 with no more detour. The existence of nonarithmetic lattices in real rank one Lie groups, however, necessitates our appealing to the more classical local rigidity theory. The latter makes weaker assumptions on $G$ at the cost of the weaker conclusion $\Gamma \subset \operatorname{GL}(n, \mathbb{Q})$. But this turns out to be good enough for our purposes.

Proof of Theorem 1. We will mostly follow [2, Proof of Proposition 5.1, p. 38]. We will prove this by induction on the dimension of the surrounding Lie group $G$. If $G$ is zero-dimensional, $\Gamma$ is a finite group and thus trivially satisfies the Farrell-Jones conjecture. Since $G$ has finitely many path components, $\Gamma \cap G^{0}$ has finite index in $\Gamma$ and is a lattice in $G^{0}$ so that we may assume that $G$ is connected by Theorem 2 (10). Arguing as in the first part of the proof of Proposition 5, we may moreover assume that $G$ has no connected, compact, normal subgroup.

Let $R$ be the radical of $G$ given by the maximal connected normal solvable subgroup of $G$. Similarly we denote by $N$ the nilradical of $G$ given by the maximal connected normal nilpotent subgroup of $G$. Clearly $N \unlhd R$ and $R / N$ is abelian. Recall from [30, Theorem 3.18.13, p. 244] that $G$ possesses maximal semisimple subgroups, any such two are conjugate, and for any such $S \subset G$ we have the generalized Levi decomposition $G=R S$. As a word of warning, in general neither $S$ nor the intersection $R \cap S$ is a closed subgroup of $G$. An example of such an $S$ is given by Alain Valette in [11.

We want to prove that $\Gamma \cap N$ is a lattice in $N$. According to [31, Theorem 1.6, p. 106] a sufficient condition is that every compact factor of $S$ acts non-trivially on $R$. Suppose $K$ was a compact factor in $S$ acting trivially on $R$. Any element $g \in G$ is of the form $g=r s$ with $r \in R$ and $s \in S$. We get for $k \in K$ that $s k s^{-1} \in$ $K$ and $\left(s k s^{-1}\right)^{-1} r\left(s k s^{-1}\right)=r$ and hence $g k g^{-1}=r\left(s k s^{-1}\right) r^{-1}=s k s^{-1} \in K$. Thus $K$ is normal in $G$ whence trivial. It follows that $\Gamma \cap N$ is a lattice in $N$. By 31, Theorem 4.7, p. 23] we conclude that $\Gamma /(\Gamma \cap N)$ is a lattice in $G / N$. We have the short exact sequence

$$
1 \rightarrow \Gamma \cap N \rightarrow \Gamma \stackrel{p}{\rightarrow} \Gamma /(\Gamma \cap N) \rightarrow 1
$$

Since $G$ has no connected, compact, normal subgroup, the nilradical is simply connected [18, Lemma 3.1.(i), p. 229]. It follows from [26, Proposition 3.7, p. 52] that the lattice $\Gamma \cap N \subset N$ is poly- $\mathbb{Z}$, i.e., polycyclic with infinite cyclic factor groups.

\footnotetext{
${ }^{1}$ Note that Theorem 4.7 in loc. cit. is obviously misprinted. The conclusion should read "... if and only if $\Gamma \cap H$ is a lattice in $H$."
} 
Hence the preimage of a virtually cyclic subgroup of $\Gamma /(\Gamma \cap N)$ under $p$ is virtually poly-ZZ as well, thus lies in $\mathfrak{F} \mathfrak{J}$ by Theorem 2 (2) and (10). Now if $N$ is non-trivial, then the Lie group $G / N$ is of lower dimension than $G$ and hence $\Gamma /(\Gamma \cap N) \in \mathfrak{F} \mathfrak{J}$ by the induction hypothesis. Therefore $\Gamma \in \mathfrak{F} \mathfrak{J}$ by Theorem 2 (9). If on the other hand $N$ is trivial, then $R \cong R / N$ is abelian, so $R$ is contained in the nilradical $N$ and thus trivial. Therefore $G$ is semisimple and Proposition 5 completes the proof.

Remark 7. According to [26, Corollary 8.28, p. 150] the criterion that no compact factor of $S$ acts trivially on $R$ is actually sufficient for $\Gamma / \Gamma \cap R$ being a lattice in $G / R$. This result would spare us the detour of factoring out the nilradical and using induction on $\operatorname{dim} G$. However, A. N. Starkov 29] claims to construct a counterexample to Corollary 8.28 , which earned him a doubtful Mathematical Review and a follow-up paper by T. S. Wu 35] counterclaiming to give a new proof of the result in question. On the other hand, E. B. Vinberg et. al. [31, p. 107] say the counterexample of Starkov is correct. We refrain from taking sides in the discussion and prefer to give our more involved but safe argument.

\section{Generalizations}

Two assumptions in Theorem 1 can still be relaxed. Firstly, the notion of lattice still makes sense for locally compact Hausdorff groups because of the existence of a unique Haar measure. Secondly, one can try and work with less restrictive connectivity. Here is the most general result we could come up with.

Theorem 8 (Lattices in second countable locally compact Hausdorff groups). Let $G$ be a second countable locally compact Hausdorff group and let $\Gamma$ be a lattice in $G$. If $\pi_{0}(G)$ is discrete and lies in $\mathfrak{F} \mathfrak{J}$, then $\Gamma$ also lies in $\mathfrak{F} \mathfrak{J}$.

We remark that the Hausdorff assumption for topological groups is often implicit in the literature. In fact a topological $T_{0}$-group is already Hausdorff. For the proof of Theorem 8 let us first recall some basic facts about the automorphism group $\operatorname{Aut}(G)$ of a connected Lie group $G$. Differentiation defines a homomorphism of Lie groups d: $\operatorname{Aut}(G) \rightarrow \operatorname{Aut}(\mathfrak{g})$ which is actually injective and has closed image [24, Proposition 4.1, p. 49]. If $G$ is simply-connected or has trivial center, then this map is an isomorphism. For the inner automorphisms $\operatorname{Int}(G)$ of $G$ we obtain $\mathrm{d}(\operatorname{Int}(G))=\operatorname{Ad}(G)$ by the very definition of the adjoint representation. As remarked in the proof of Proposition 5 we have $\operatorname{Ad}(G)=\operatorname{Int}(\mathfrak{g})$ so that d induces an injective group homomorphism $\operatorname{Out}(G) \rightarrow \operatorname{Out}(\mathfrak{g})$ where $\operatorname{Out}(G)=\operatorname{Aut}(G) / \operatorname{Int}(G)$ and $\operatorname{Out}(\mathfrak{g})=\operatorname{Aut}(\mathfrak{g}) / \operatorname{Int}(\mathfrak{g})$ denote the groups of outer automorphisms. If $G$ is moreover semisimple, then we have seen in the same proof that $\mathbf{G}=\operatorname{Aut}(\mathfrak{g})$ is a linear algebraic $\mathbb{Q}$-group with $\mathbf{G}(\mathbb{R})^{0}=\operatorname{Int}(\mathfrak{g})$. By a theorem of Whitney 34, Theorem 3, p. 547] a real algebraic variety has only finitely many components in the ordinary topology. Applying this result to the $\mathbb{R}$-variety $\mathbf{G}(\mathbb{R})$ we have come to the following conclusion.

Lemma 9. The group of outer automorphisms Out $(G)$ of a connected semisimple Lie group $G$ is finite.

We use this fact to draw the following conclusion.

Proposition 10. Let $\Gamma$ be a lattice in a connected Lie group $G$, and let $\varphi$ be an automorphism of $G$ with $\varphi(\Gamma)=\Gamma$. Then $\Gamma \rtimes_{\varphi} \mathbb{Z}$ lies in $\mathfrak{F} \mathfrak{J}$.

Proof. We will prove this by induction on the dimension of $G$. The induction beginning $\operatorname{dim}(G)=0$ is trivial; in this case $G$ and hence $\Gamma$ are trivial so that $\Gamma \rtimes \mathbb{Z} \cong \mathbb{Z}$ and hence satisfies the Farrell-Jones conjecture for trivial reasons. 
First we want to reduce the general case to the case of a lattice in a semisimple Lie group. This works similarly as in the proof of Theorem 11 The nilradical $N$ of $G$ is characteristic, therefore $\varphi$-invariant and hence we get a short exact sequence.

$$
1 \rightarrow \Gamma \cap N \rightarrow \Gamma \rtimes_{\varphi} \mathbb{Z} \rightarrow \Gamma /(\Gamma \cap N) \rtimes \mathbb{Z} \rightarrow 1
$$

As we have seen above, the group $\Gamma \cap N$ is a lattice in $N$ and polycyclic. Thus preimages of virtually cyclic subgroups are virtually polycyclic and hence they lie in $\mathfrak{F} \mathfrak{J}$ by Theorem 2 (2) and (10). If $N$ is non-trivial, it has dimension bigger than zero and thus $G / N$ has smaller dimension. Hence the lattice $\Gamma /(\Gamma \cap N) \rtimes \mathbb{Z}$ lies in $\mathfrak{F} \mathfrak{J}$ by induction assumption and so does $\Gamma \rtimes_{\varphi} \mathbb{Z}$ by Theorem 2(9). If $N$ is trivial, then the radical $R$ is also trivial which means that $G$ is additionally semisimple.

Let $K$ be the maximal compact factor of $G$. It is likewise characteristic and hence $\varphi$-invariant. Thus we have a short exact sequence

$$
1 \rightarrow \Gamma \cap K \rightarrow \Gamma \rtimes_{\varphi} \mathbb{Z} \stackrel{p}{\rightarrow} \Gamma /(\Gamma \cap K) \rtimes_{\varphi} \mathbb{Z} \rightarrow 1
$$

Since $K \cap \Gamma$ is finite, any preimage of a virtually cyclic subgroup under $p$ is again virtually cyclic and hence it lies in $\mathfrak{F} \mathfrak{J}$. Thus by Theorem 2(9) it remains to show the statement for $\Gamma /(\Gamma \cap K) \rtimes_{\varphi} \mathbb{Z}$. As explained above $\Gamma /(\Gamma \cap K)$ is a lattice in the Lie group $G / K$. If $K$ is non-trivial, then the target has smaller dimension and thus satisfies the Farrell-Jones conjecture by induction assumption. It remains to consider the case where $G$ is semisimple without compact factors.

The outer automorphism group of $G$ is finite by Lemma9. Since $\Gamma \rtimes_{\varphi^{n}} \mathbb{Z}$ has finite index in $\Gamma \rtimes_{\varphi} \mathbb{Z}$, we can use Theorem 2 (10) to replace $\varphi$ by a power of $\varphi$ and thus we may assume that $\varphi$ is given by conjugation with $g \in G$. By [17, Corollary 2.2, p. 313] we have that the Weyl group $\left\{g \in G \mid g \Gamma g^{-1}=\Gamma\right\} / \Gamma$ is finite. Thus after further passing to a power we may assume that $\varphi$ is given by conjugation with $\gamma \in \Gamma$. Thus $\Gamma \rtimes_{\varphi} \mathbb{Z} \cong \Gamma \times \mathbb{Z}$. The isomorphism is given by the identity on $\Gamma$ and it sends the generator of $\mathbb{Z} \subset \Gamma \rtimes_{\varphi} \mathbb{Z}$ to $(\gamma, 1) \in \Gamma \times \mathbb{Z}$. Hence $\Gamma \rtimes_{\varphi} \mathbb{Z}$ lies in $\mathfrak{F} \mathfrak{J}$ by Theorem 2 (7) and Proposition 5

Remark 11. We did not show the Farrell-Jones conjecture for all groups of the form $\Gamma \rtimes \mathbb{Z}$, where $\Gamma$ is a lattice in a connected Lie group. We only showed this for those automorphisms which extend to an automorphism of the surrounding Lie group. Nevertheless, in interesting cases these extensions always exist and are unique, most notably if $\Gamma$ is an irreducible lattice in a connected semisimple Lie group without compact factors, with trivial center and not isomorphic to $\operatorname{PSL}(2, \mathbb{R})[23$, Theorem 7.5 and Remark 7.6, p. 254]. Note that the last requirement is essential because the group $\operatorname{PSL}(2, \mathbb{Z})$ contains the free group on three letters $F_{3}$ as a subgroup of index twelve, so $F_{3}$ is a lattice in $\operatorname{PSL}(2, \mathbb{R})$. If unique extension of automorphisms held for lattices in $\operatorname{PSL}(2, \mathbb{R})$, we would obtain an embedding $\operatorname{Aut}\left(F_{3}\right) \rightarrow \operatorname{Aut}(\mathfrak{s l}(2, \mathbb{R})) \subset \mathrm{GL}(3, \mathbb{R})$. It is however well-known that $\operatorname{Aut}\left(F_{3}\right)$ has no faithful linear representation [15.

Theorem 12. Let $\Gamma$ be a lattice in a Lie group $G$. If $\pi_{0}(G)$ lies in $\mathfrak{F} \mathfrak{J}$, so does $\Gamma$.

Proof. Let $p: G \rightarrow \pi_{0}(G)$ be the projection. Its kernel $G^{0}$ is the path component of the identity. We get an induced group homomorphism $\Gamma \rightarrow p(\Gamma)$ whose target lies in $\mathfrak{F} \mathfrak{J}$. We want to apply Theorem 2 (9). Since $G^{0}$ is open, the path components of $G / \Gamma$ have positive measure. Therefore the index of $p(\Gamma)$ in $\pi_{0}(G)$ must be finite. Since $G^{0}$ is closed, [31, Theorem 4.7, p. 23] asserts that $\Gamma \cap G^{0}$ is a lattice in $G^{0}$. (Mind the footnote on p. 5.) So the group $\Gamma \cap G^{0}$ lies in $\mathfrak{F} \mathfrak{J}$ by Theorem 1 Thus it remains to check that the preimage of every infinite cyclic subgroup $Z$ of $p(\Gamma)$ lies 
in $\mathfrak{F} \mathfrak{J}$. We have short exact sequences

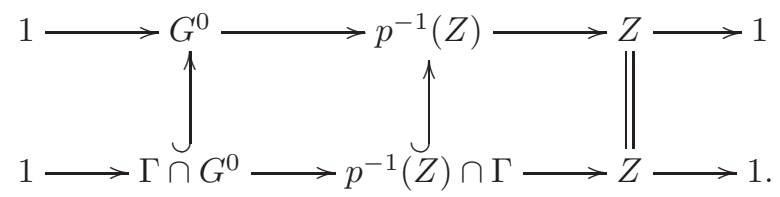

Thus $p^{-1}(Z) \cap \Gamma$ can be expressed as a semidirect product $G^{0} \cap \Gamma \rtimes_{\varphi} \mathbb{Z}$. The automorphism $\varphi$ is given by conjugation with a preimage $\gamma \in p^{-1}(Z) \cap \Gamma$ of a generator of $Z$. Thus we can apply Proposition 10 to the lattice $\Gamma \cap G^{0}$ in $G^{0}$ with the automorphism $\varphi$.

Proof of Theorem 8. Let $K$ be the unique maximal, compact, normal subgroup of $G^{0}$. The Montgomery-Zippin solution to Hilbert's fifth problem implies that the factor group $G^{0} / K$ is a Lie group, see for instance [1, Lemma 1, p. 274]. By uniqueness $K$ is characteristic in $G^{0}$ and thus normal in $G$. Consequently $G / K$ is homeomorphic to the disjoint union of $\pi_{0}(G)$-copies of $G^{0} / K$. The discrete space $\pi_{0}(G)$ is countable because $G$ is second countable, so in fact $G / K$ is a Lie group as well. Moreover $\pi_{0}(G / K)=\pi_{0}(G)$. As in the beginning of the proof of Proposition 5 , the group $\Gamma / \Gamma \cap K$ is a lattice in $G / K$ and $\Gamma / \Gamma \cap K$ lies in $\mathfrak{F} \mathfrak{J}$ if and only if $\Gamma$ does. Theorem 12 completes the proof.

\section{REFERENCES}

[1] R. W. Bagley and M. R. Peyrovian, A note on compact subgroups of topological groups, Bull. Austral. Math. Soc. 33 (1986), no. 2, 273-278, DOI 10.1017/S0004972700003142 MR 832529

[2] A. Bartels, T. Farrell, and W. Lück, The Farrell-Jones Conjecture for cocompact lattices in virtually connected Lie groups (2013), eprint, to appear in Journal of the AMS. arXiv:1101.0469v2

[3] A. Bartels and W. Lück, On crossed product rings with twisted involutions, their module categories and L-theory, Cohomology of groups and algebraic $K$-theory, Adv. Lect. Math. (ALM), vol. 12, Int. Press, Somerville, MA, 2010, pp. 1-54 MR 2655174

[4] , The Borel conjecture for hyperbolic and CAT(0)-groups, Ann. of Math. (2) 175 (2012), no. 2, 631-689, DOI 10.4007/annals.2012.175.2.5/MR 2993750

[5] A. Bartels, W. Lück, and H. Reich, On the Farrell-Jones conjecture and its applications, J. Topol. 1 (2008), no. 1, 57-86, DOI 10.1112/jtopol/jtm008/MR 2365652

[6] (2008), no. 1, 29-70, DOI 10.1007/s00222-007-0093-7 MR 2385666

[7] A. Bartels, W. Lück, H. Reich, and H. Rüping, $K$ - and $L$ - theory of group rings over $\operatorname{GL}(n, \mathbb{Z})$, Publications mathématiques de l'IHÉS (2013). online version available at http://link.springer.com/article/10.1007\%2Fs10240-013-0055-0

[8] A. Bartels and H. Reich, Coefficients for the Farrell-Jones conjecture, Adv. Math. 209 (2007), no. 1, 337-362, DOI 10.1016/j.aim.2006.05.005 MR 2294225

[9] A. Borel, Compact Clifford-Klein forms of symmetric spaces, Topology 2 (1963), 111122 MR 0146301

[10] M. R. Bridson and A. Haefliger, Metric spaces of non-positive curvature, Grundlehren der Mathematischen Wissenschaften [Fundamental Principles of Mathematical Sciences], vol. 319, Springer-Verlag, Berlin, 1999 MR 1744486

[11] Yemon Choi, Are maximal connected semisimple subgroups automatically closed? URL http://mathoverflow.net/q/118505 (version: 2013-01-10).

[12] P. Deligne and G. D. Mostow, Monodromy of hypergeometric functions and non-lattice integral monodromy, Inst. Hautes Études Sci. Publ. Math. 63 (1986), 5-89 MR 849651

[13] F. T. Farrell and L. E. Jones, Isomorphism conjectures in algebraic K-theory, J. Amer. Math. Soc. 6 (1993), no. 2, 249-297, DOI 10.2307/2152801 MR 1179537

[14] F. T. and Jones Farrell L. E., Rigidity for aspherical manifolds with $\pi_{1} \subset G L_{m}(\mathbb{R})$, Asian J. Math. 2 (1998), no. 2, 215-262.

[15] E. Formanek and C. Procesi, The automorphism group of a free group is not linear, J. Algebra 149 (1992), no. 2, 494-499, DOI 10.1016/0021-8693(92)90029-L/MR 1172442

[16] H. Garland and M. S. Raghunathan, Fundamental domains for lattices in (R-)rank 1 semisimple Lie groups, Ann. of Math. (2) 92 (1970), 279-326 MR 0267041 
[17] F. P. Greenleaf and M. Moskowitz, Finiteness results for lattices in certain Lie groups, Ark. Mat. 48 (2010), no. 2, 311-321, DOI 10.1007/s11512-009-0112-6. MR 2672612

[18] F. P. Greenleaf, M. Moskowitz, and L. P. Rothschild, Unbounded conjugacy classes in Lie groups and location of central measures, Acta Math. 132 (1974), 225-243 MR 0425035

[19] S. Helgason, Differential geometry, Lie groups, and symmetric spaces, Graduate Studies in Mathematics, vol. 34, American Mathematical Society, Providence, RI, 2001. Corrected reprint of the 1978 original MR 1834454

[20] W. Lück, $K$ - and L-theory of group rings, Proceedings of the International Congress of Mathematicians. Volume II, Hindustan Book Agency, New Delhi, 2010, pp. 1071-1098 MR 2827832

[21] W. Lück and H. Reich, The Baum-Connes and the Farrell-Jones conjectures in K- and $L$-theory, Handbook of $K$-theory. Vol. 1, 2, Springer, Berlin, 2005, pp. 703-842 MR 2181833

[22] G. A. Margulis, Arithmeticity of the irreducible lattices in the semisimple groups of rank greater than 1, Invent. Math. 76 (1984), no. 1, 93-120, DOI 10.1007/BF01388494 MR 739627

[23] - Discrete subgroups of semisimple Lie groups, Ergebnisse der Mathematik und ihrer Grenzgebiete (3) [Results in Mathematics and Related Areas (3)], vol. 17, Springer-Verlag, Berlin, 1991.MR 1090825

[24] A. L. Onishchik, Lie groups and Lie algebras. I, Encyclopaedia of Mathematical Sciences, vol. 20, Springer-Verlag, Berlin, 1993. Foundations of Lie theory. Lie transformation groups; A translation of Current problems in mathematics. Fundamental directions. Vol. 20 (Russian), Akad. Nauk SSSR, Vsesoyuz. Inst. Nauchn. i Tekhn. Inform., Moscow, 1988 [MR 0950861; Translation by A. Kozlowski; Translation edited by A. L. Onishchik MR 1306737

[25] V. Platonov and A. Rapinchuk, Algebraic groups and number theory, Pure and Applied Mathematics, vol. 139, Academic Press Inc., Boston, MA, 1994. Translated from the 1991 Russian original by Rachel Rowen MR 1278263

[26] M. S. Raghunathan, Discrete subgroups of Lie groups, Springer-Verlag, New York, 1972. Ergebnisse der Mathematik und ihrer Grenzgebiete, Band 68 MR 0507234

[27] D. L. Ragozin, A normal subgroup of a semisimple Lie group is closed, Proc. Amer. Math. Soc. 32 (1972), 632-633 MR 0294563

[28] H. Rüping, The Farrell-Jones conjecture for S-arithmetic groups (2013), eprint available at. arXiv:1309.7236v1

[29] A. N. Starkov, A counterexample to a theorem on lattices in Lie groups, Vestnik Moskov. Univ. Ser. I Mat. Mekh. 5 (1984), 68-69 (Russian) MR 764036

[30] V. S. Varadarajan, Lie groups, Lie algebras, and their representations, Prentice-Hall Inc., Englewood Cliffs, N.J., 1974. Prentice-Hall Series in Modern Analysis MR 0376938

[31] E. B. Vinberg, V. V. Gorbatsevich, and O. V. Shvartsman, Discrete subgroups of Lie groups MR 0968445], Lie groups and Lie algebras, II, Encyclopaedia Math. Sci., vol. 21, Springer, Berlin, 2000, pp. 1-123, 217-223 MR 1756407

[32] C. Wegner, The K-theoretic Farrell-Jones conjecture for CAT(0)-groups, Proc. Amer. Math. Soc. 140 (2012), no. 3, 779-793, DOI 10.1090/S0002-9939-2011-11150-X MR 2869063

[33] _ The Farrell-Jones Conjecture for virtually solvable groups (2013), eprint available at. arXiv:1308.2432v2

[34] H. Whitney, Elementary structure of real algebraic varieties, Ann. of Math. (2) 66 (1957), 545-556 MR 0095844

[35] T. S. Wu, A note on a theorem on lattices in Lie groups, Canad. Math. Bull. 31 (1988), no. 2, 190-193, DOI 10.4153/CMB-1988-029-8. MR 942071

Mathematisches Institut, Universität Bonn, Endenicher Allee 60, 53115 Bonn, GerMANY

E-mail address: kammeyer@math.uni-bonn.de

URL: http://www.math.uni-bonn.de/people/kammeyer/

E-mail address: wolfgang.lueck@him.uni-bonn.de

$U R L:$ http://www.him.uni-bonn.de/lueck/

E-mail address: henrik.rueping@hcm.uni-bonn.de

$U R L$ : http://www .math.uni-bonn.de/people/rueping/ 